54, 1, pp. 195-204, Warsaw 2016

DOI: $10.15632 /$ jtam-pl.54.1.195

\title{
POST-CRITICAL DEFORMATION STATES OF COMPOSITE THIN-WALLED AIRCRAFT LOAD-BEARING STRUCTURES
}

\author{
Tomasz Kopecki, Jerzy Bakunowicz, Tomasz Lis \\ Rzeszów University of Technology, Faculty of Mechanical Engineering and Aeronautics, Rzeszów, Poland \\ e-mail: tkopecki@prz.edu.pl; bakun@prz.edu.pl; list@prz.edu.pl
}

\begin{abstract}
The study presents results of experimental examination of a model representing a fragment of an aircraft wing structure with the skin made of a glass fibre/epoxy composite. For such a system, the deformation pattern has been found and the representative equilibrium path determined. The finite element method has been used to develop the corresponding numerical model, the correctness of which has been then verified by comparing the obtained results with the course of the relevant experiment. Conformity of the results allowed one to determine usefulness of the applied methods in the assessment of mechanical properties of modified solutions involving integral skin stiffening elements.
\end{abstract}

Keywords: skin, loss of stability, finite element method, composite, equilibrium path

\section{Introduction}

Contemporary aircraft structures represent a group of devices that are, and, for many years have been invariably expected to meet the highest requirements in the area of their properties, fulfil exorbitant economical criteria and comply with particularly rigorous safety-related rules. To meet the latter, design engineers are compelled to continuously improve the design processes the fundamental portion of which is nowadays realised in the space of virtual objects by using sophisticated numerical tools. Among a number of software types utilised commonly in various stages of designing and fabrication of new structures, a specially important role should be attributed to programs based on the finite element method (FEM).

One of the problems related to application of such programs that, despite continuous progress in the aircraft technology, still lacks the ultimate solution, consists in imperfections of the utilised numerical models and the resulting divergences between the results obtained by means of them and behaviour of actual objects (Ramm and Wall, 2004). While the general knowledge and available databases containing results of experimental research for typical isotropic materials used in the aviation industry, such as e.g. titanium and aluminium alloys, allow one to define the above-mentioned models with sufficient precision, the structures based on composites of various types, although utilised more and more frequently, are still particularly troublesome objects to analyse (Seresta, 2007). It follows from the fact that results pertaining to experimental studies on composite structures, especially those dealing with their behaviour under cyclic load conditions and at occurrence of large deformations, are in general considered trade secrets of aerospace corporations and are disclosed only in a fragmentary scope in commonly available scientific publications.

A feature that is highly distinctive for aircraft structures in comparison with other groups of thin-walled systems is the admissibility of occurrence of post-critical deformation in their elements (Dow et al., 1954), provided that the related loss of stability has an elastic nature and occurs locally within the area of skin segments limited by components of the skeleton (Taylor and Eckford, 1968). The principle is used commonly with respect to metal structures (Niu, 1988). 
In the case of composite structures, the rule applicable for many years provided the obligatory use of solutions making the occurrence of skin buckling impossible, e.g. by means of using interlayers in the form of foamed plastics or cellular cores (Federal Aviation Administration, 2009). The above-mentioned rule in combination with the stiffness-based composite structure designing criterion can be applied to light aircraft structures (gliders, light-sports aircraft), but it must be considered inapplicable to extensive structures such as wings of large transportation aeroplanes in which large deformations are inevitable. In the process of designing contemporary transportation aircraft, in aspiration to minimise the overall mass of the structure, the use of composite skins are becoming more and more popular among designers constructing load-bearing demanding elements in the case of which a local stability loss is admissible. Examples of such solutions can be found in such aeroplanes as American Boeing 787 and European Airbus 350. In the case of the former, the main cause of delay in realisation of the project were problems with finding an appropriate design solution for the composite bearing structure. The scale of research projects necessary to realise undertakings of that scope can be partially illustrated by the input provided by projects POSICOSS and COCOMAT financed within the European Commission Framework Programme. However, there is always a serious fear that as a result of bending, relative high stress gradients may appear in outer layers of the skin that in turn may become a cause of its premature destruction (Arborcz, 1985; Nemeth, 2013).

Application of the above design principle involves therefore absolute necessity to perform experiments aimed at precise determination of mechanical properties of individual components of the structure in cyclic post-critical deformation conditions (Kopecki, 2019; Kopecki and Mazurek, 2013). It should be noted at this point that the results obtained in the case of composites may depend both on the skin thickness resulting from the number of fabric layers and the manufacturing process, type and properties or components as well as and fibre orientation. In view of multitude of such factors having the effect on the structure properties, the possibility to generalise experimental results concerning mechanical properties of composites is very limited.

Regardless of the composite type, advisability to strive after limitation of magnitude of postcritical deformations occurring in operating conditions seems to be obvious. Solutions aspiring to obtain such a target include employment of integral stiffening elements of various types as well as changing proportions and reducing dimensions of skin segments at risk of loosing stability.

Realisation of relevant experiments with the use of models representing selected variants of solutions of that type can constitute a basis for development and making more specific numerical models formulated in terms of the finite element method thanks to which it would be possible to determine effects of a number of further modifications of the examined structures.

\section{Purpose and scope of the research}

Considerations presented in this study represent the first stage of a planned series of experimental-numerical analyses of thin-walled composite aircraft structures. The research program is aimed at determination of the effect of selected variants of integral skin stiffening elements on the critical load value and post-critical deformation magnitude in combination with mass analysis, the effect of which should be a structure with the most effective total mass/durability ratio.

The research stage described in this paper involves realisation of an experiment with the use of a model of a representative aircraft structure, and then the development of an adequate numerical model of the structure the nonlinear analysis of which, carried out with the use of the finite element method, would allow one to obtain a distribution of deformations corresponding to actual ones both qualitatively and quantitatively. 
The subject of the analysis was a fragment of a monospar wing structure with a constant chord along the whole span and the stiffened front torsion box (Fig. 1). The examined variant constituted the simplest possible system of that type and lacked any integrated longitudinal skin stiffening elements, playing the role of a reference structure for subsequent variants with modified geometry and stiffness planned to be examined.

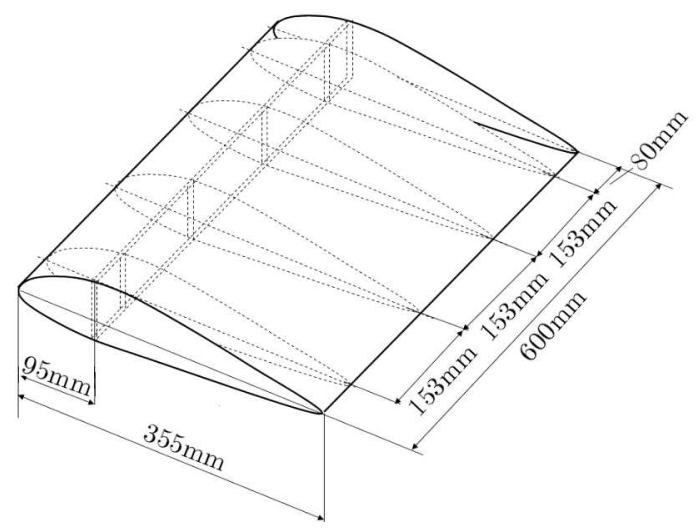

Fig. 1. An overall outline of the examined structure

The model was manufactured in the wet lay-up method and handmade formed in moulds. The composite consisted of Interglass 02037 and 92110 glass fabrics and MGS L285/H286 epoxy resin and was characterised by $E_{11}=22000 \mathrm{MPa}, E_{22}=22000 \mathrm{MPa}, \nu_{1} 2=0.11, G_{12}=4600$ constants. The upper and lower wing surfaces were connected to the spruce box spar and plywood web of $1 \mathrm{~mm}$ thickness. Additionaly, the roots were reinforced by plywood ribs with joints. The whole wing was manufactured from two halves attached together in mouldings and then cured in the LPC process.

\section{Experimental research}

The skeleton portion of the model used in the experiment was made of plywood and wooden slats with known mechanical parameters. The skin was an epoxy composite reinforced with glass fibre (GFRP).

As the composite reinforcement, Interglass glass fibre fabrics were used with the weight ratios of $50 \mathrm{~g} / \mathrm{m}^{2}$ and $163 \mathrm{~g} / \mathrm{m}^{2}$. The matrix was a permeating mix based on epoxy resin MGSL285. The skin of the model in the torsion box zone was made as a laminar structure comprising three layers of a symmetric fabric. The main directions of the composite ortotrophy were oriented at the angle of 45 degrees with respect to the direction of the spar flanges. The remaining portion of the structure contained two layers of the fabric (Fig. 2). Such a solution was aimed at protecting the torsion box surface against the loss of stability and creation of conditions favourable to the occurrence of post-critical deformations in the skin part between the spar and the trailing edge. The reinforcement coefficient of the composite was $50 \%$ in mass. The "mother" specimen for each composite type was fabricated in the same process, additionally. Analysis of the specimen weight allowed one to calculate the reinforcement coefficient, knowing weight of the fabric prior to fabrication.

In the course of experiment, the model was subjected to simultaneous bending and torsional deflection (Fig. 3) on a specially constructed experimental set-up (Fig. 4). Influence of friction in the bearing roller was neglected.

The load was applied gravitationally. In the course of the experiment, displacements of selected reference points were measured for subsequent stationary deformation states of the structure. 


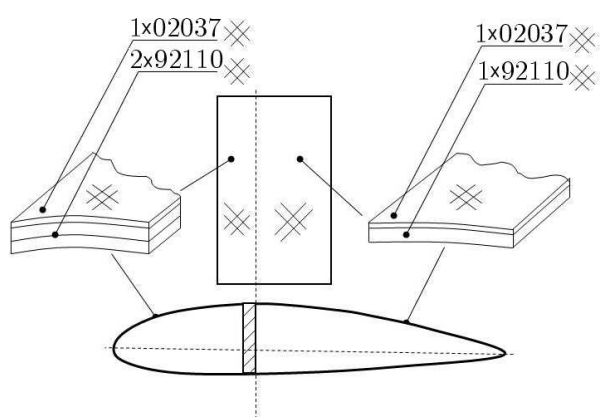

Fig. 2. Schematic representation of lamination of the considered structure
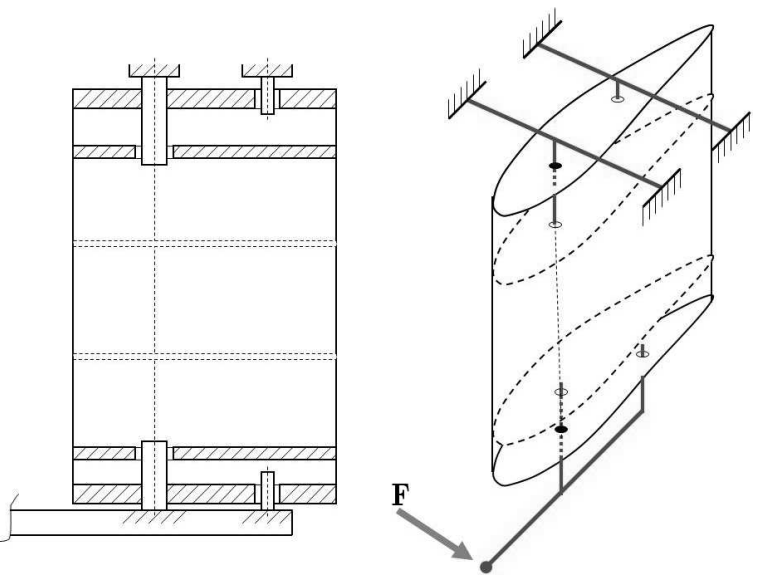

Fig. 3. An outline of the model fastening and load application (TL)

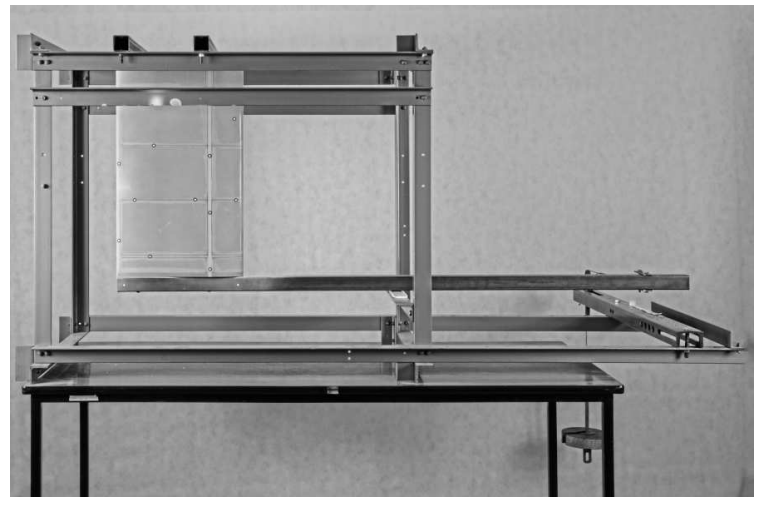

Fig. 4. The research set-up

(a)

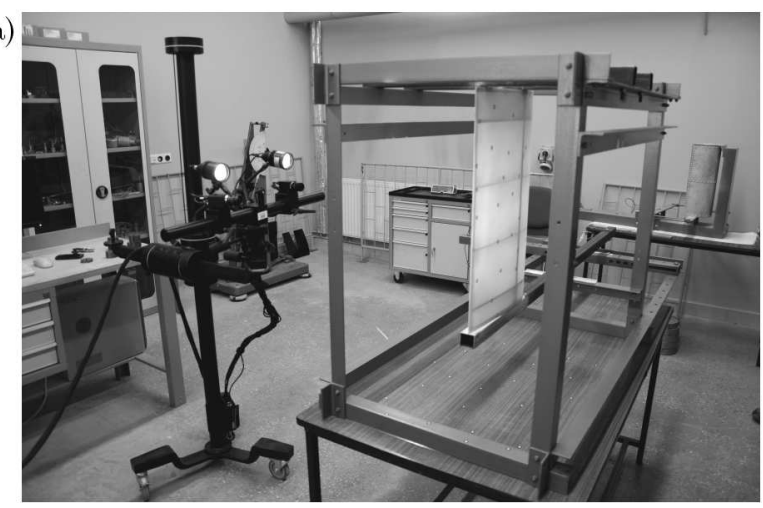

(b)

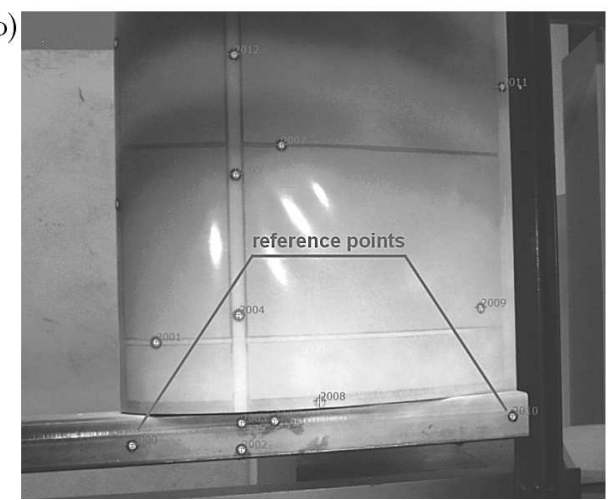

Fig. 5. (a) PONTOS measuring system; (b) selected reference points 
To measure the displacements, PONTOS measuring system by GOM Optical Measuring Techniques was used (Fig. 5). As a result, a representative equilibrium path was obtained constituting a relationship between the structure total torsion angle and the load value (Fig. 6).

(a)

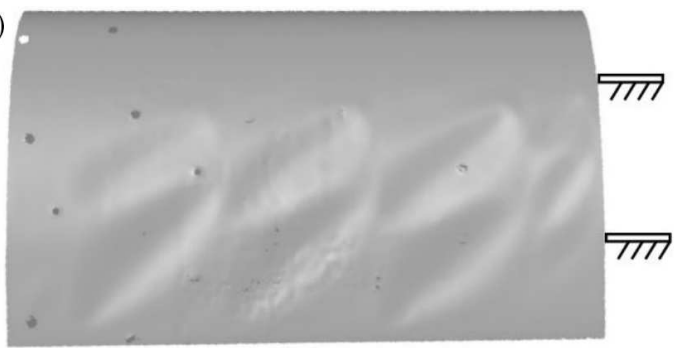

(b)

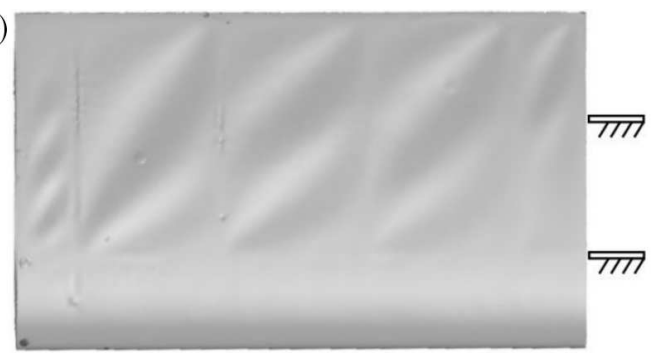

Fig. 6. Model deformations determined by means of ATOS scanner; (a) upper skin, (b) lower skin

Moreover, for the target load value, the whole surface of the deformed model was scanned with the use of ATOS scanner. As a result, the deformation fields was obtained (Fig. 6) constituting the base for qualitative verification of the results obtained numerically.

\section{Numerical analyses}

During the computational stage of the study the necessary question to be answered was, with the nature of deformations taken into account, the possibility of developing an adequate numerical model of a composite structure with the use of commercially available software. The decisive phase of the model creation stage is the use of an algorithm the purpose of which is to determine properties of the laminate based on sets of constants corresponding to its individual layers. In the case of MSC PATRAN/MARC software used in this study, any interference of the user to the structure of the program is not possible.

A distinctive feature of composite structures making the development of their numerical representation very difficult is their inhomogeneity resulting not only from conditions in which individual layers are laminated but also from assembling operations, i.e. presence of local excesses of resin and diversified thickness of bonded joints. Such factors can induce local skin stiffness variations and have an effect on post-critical deformation patterns. Even small errors in selection of geometric parameters for the numerical model, introducing definite deviations from actual boundary conditions characterising a skin segment, generate significant errors in the course of nonlinear analyses.

The fundamental relationship in the numerical problem determining quantitatively the link between conditions of a structure and the load applied to it is the so-called equilibrium path of the system in question constituting, in general, a hypersurface in the hyperspace of states (Ramm, 1987). The relationship fulfils the matrix equation of residual forces (Felippa, 1976)

$$
\mathbf{r}(\mathbf{u}, \Lambda)=\mathbf{0}
$$

where $\mathbf{u}$ is the state vector containing components of displacements of nodes of the structure corresponding to its current geometrical configuration, $\Lambda$ is the control parameter corresponding to the current load level, and $\mathbf{r}$ is the residual vector containing non-balanced force components related to the current system deformation state. The set of control parameters can be represented by a single parameter being a function of the load. Equation (4.1) takes then the form

$$
\mathbf{r}(\mathbf{u}, \lambda)=\mathbf{0}
$$

called the single-parameter equation of residual forces. 
The prediction-correction methods of determining the consecutive points of the equilibrium path used in contemporary software routines contain also a correction phase based on the requirement that the system satisfies an additional equation called the increment control equation or the constraints equation (Bathe, 1996; Kopecki and Mazurek, 2014)

$$
c\left(\Delta \mathbf{u}_{n}, \Delta \lambda_{n}\right)=0
$$

where the increments

$$
\Delta \mathbf{u}_{n}=\mathbf{u}_{n+1}-\mathbf{u}_{n} \quad \Delta \lambda_{n}=\lambda_{n+1}-\lambda_{n}
$$

correspond to transition from the state $n$ to the state $n+1$.

In view of the lack of possibility to represent equilibrium paths for systems with more than 2 degrees of freedom in a form of easily readable plots, in practice, for the purpose of comparison, the so-called representative equilibrium paths are used which represent a functional relationship between a selected parameter characterising deformation of the system and a single control parameter related to the applied load. Reliability of results obtained from FEM-based nonlinear numerical analyses is usually accepted when a satisfactory convergence is found between two representative equilibrium paths, namely the actual one determined in the course of an experiment and that obtained numerically. It is also necessary to obtain convergence between the forms of deformations following from calculations (Ramm and Wall, 2004) with the results of a corresponding experiment. On the grounds of the solution uniqueness rule, according to which a specific deformation pattern may correspond to one and only one stress distribution pattern, the reliability can be then attributed also to the reduced stress distributions in the deformed skin (Marcinowski, 1999).

As the nonlinear numerical analysis is an iterative process aimed at finding successive equilibrium states, its correctness is to a large degree determined by correct choice of the prognostic method, the correction strategy, and a number of control parameters. In the case described here, the Newton-Raphson method has been used in combination with the Crisfield hyperspherical correction strategy.

By contrast with numerical analyses where the goal is to obtain the number of finite elements as high as possible, the use of an excessively dense grid of elements in nonlinear analyses leads sometimes to faulty results with the calculation time becoming significantly longer. After a series of numerical tests aimed at selection of a proper topology of the model, it has been decided to use a model comprising 4838 bilinear, four-node shell elements. The necessity to employ such an element resulted from the fact that other types available in the MSC MARC software library, to which the properties of laminated composites could be assigned, do not offer the possibility to reproduce geometrically complex objects, in view of the type and number of degrees of freedom.

Mechanical properties of the numerical model have been taken from the experimental data set, as decribed in Section 3. Boundary conditions of the numerical model have been simulated by fixed degrees of freedom in certain nodes of the root rib. Moreover, the loading has been simulated by a relevant beam, as in the experimental setup (Fig. 7).

The first version of the model, reproducing faithfully geometry of the actual object, turned out to lead to an incorrect form of post-critical deformations despite appropriate selection of the set of nodes and correct application of the load (Fig. 8).

In the case of the obtained results, although the representative equilibrium path of the system proved to be satisfactorily similar to this determined in the course of the experiment, it was no longer possible to rely on the above-mentioned solution uniqueness rule.

Usually, the reasons of incorrect determination of deformation pattern corresponding to the minimum energy states can be usually sought in excessively idealised reproduction of geometrical parameters of the structure. The encountered problem can be attributed to the above-discussed 


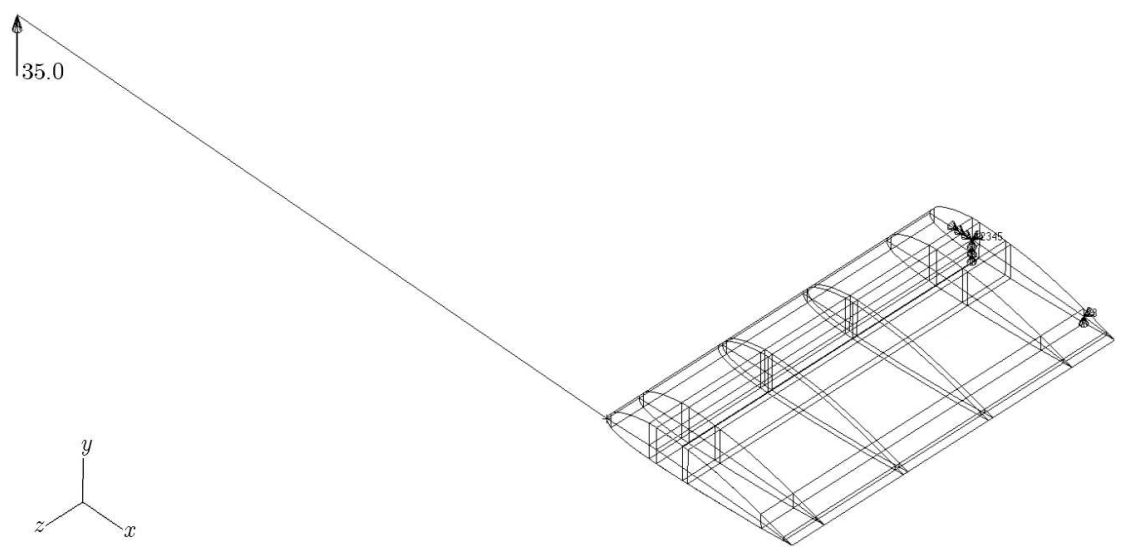

Fig. 7. Boundary conditions and load application of the numerical model

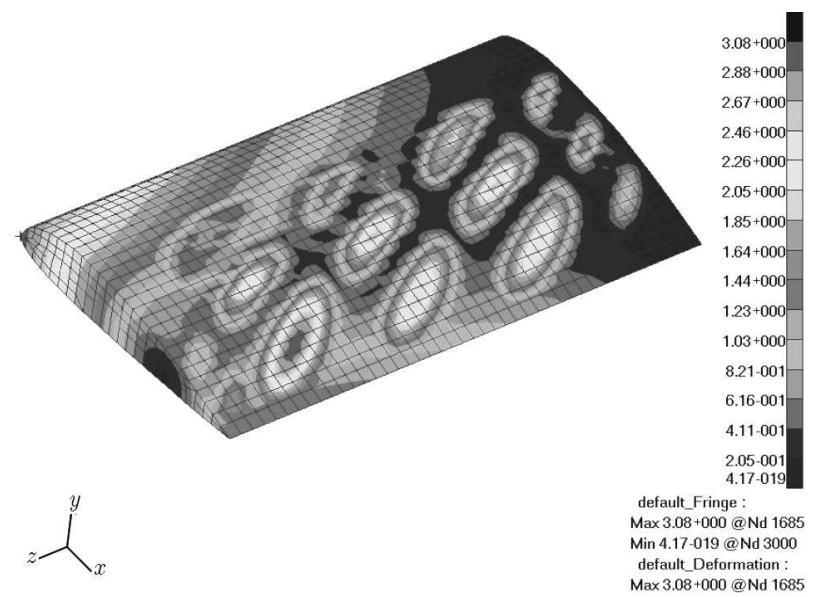

Fig. 8. Resultant displacement distribution in the first version of the numerical model found to be inconsistent with real behaviour (in $\mathrm{mm}$ )

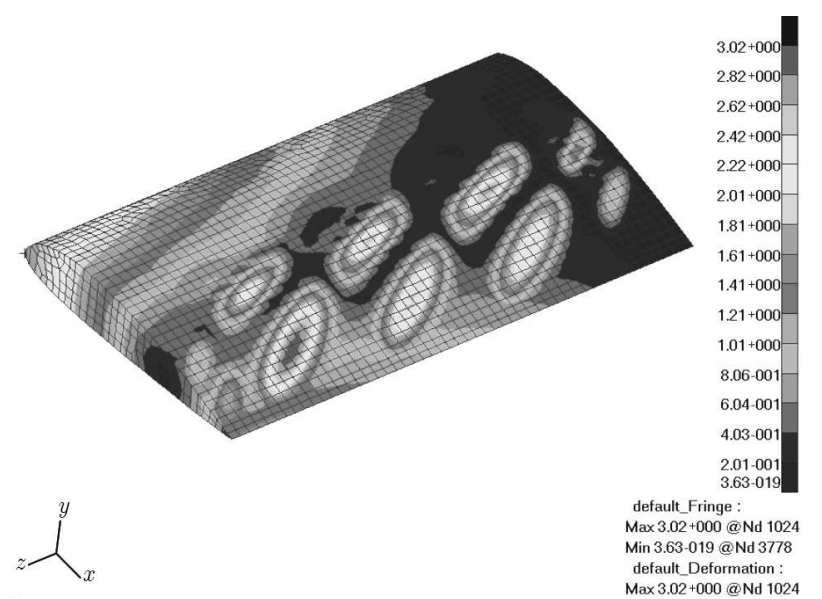

Fig. 9. Resultant displacement distribution in the corrected version of the model - the upper surface (in $\mathrm{mm}$ )

issue of geometrical inhomogeneity of the real object. In the analysed case, it turned out that the source of error consisted in too low stiffness of the rear fragments of the skin located in the vicinity of the spar. In the actual object, the feature resulting in additional stiffening of this fragment of the structure is the epoxy resin joint connecting the spar with the skin and contributing to a local increase in skin thickness. 

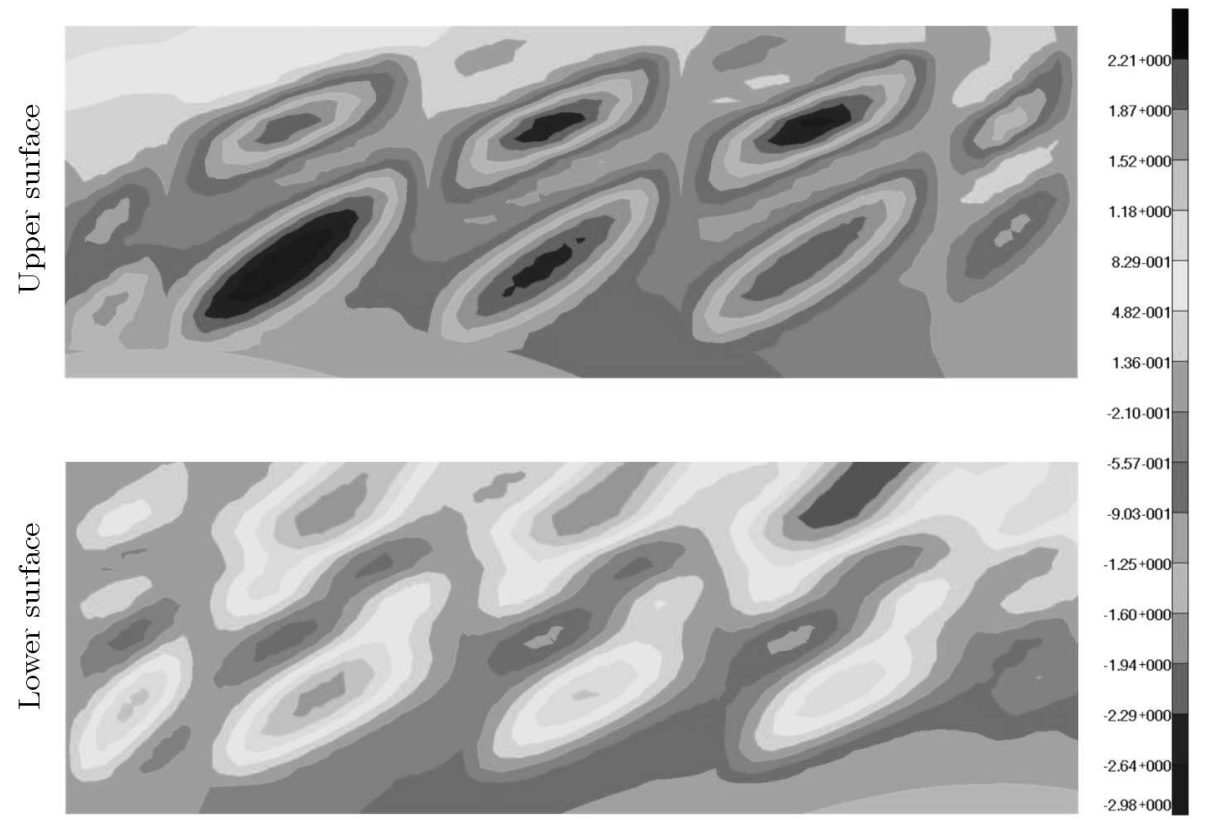

Fig. 10. Displacements in the direction normal to the surface - the skin area between the girder and the trailing edge (in $\mathrm{mm}$ )
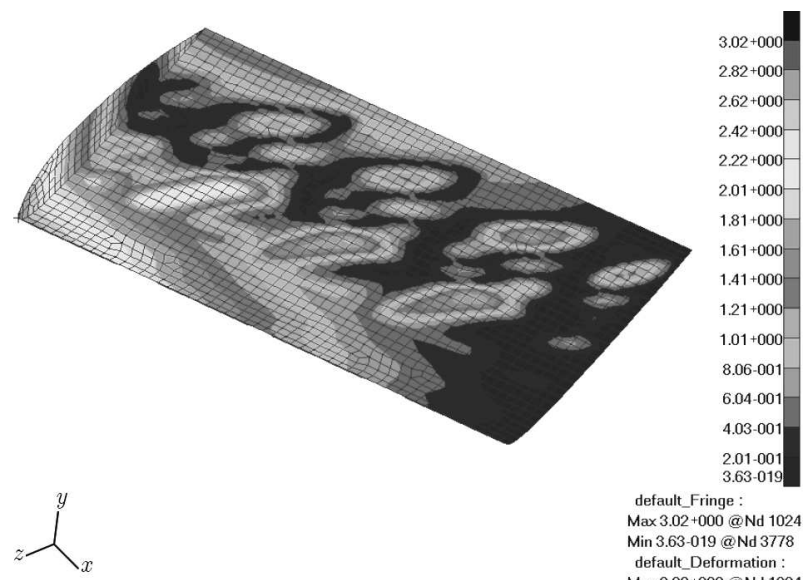

Fig. 11. Resultant displacement distribution in the corrected version of the model - the lower surface (in $\mathrm{mm}$ )

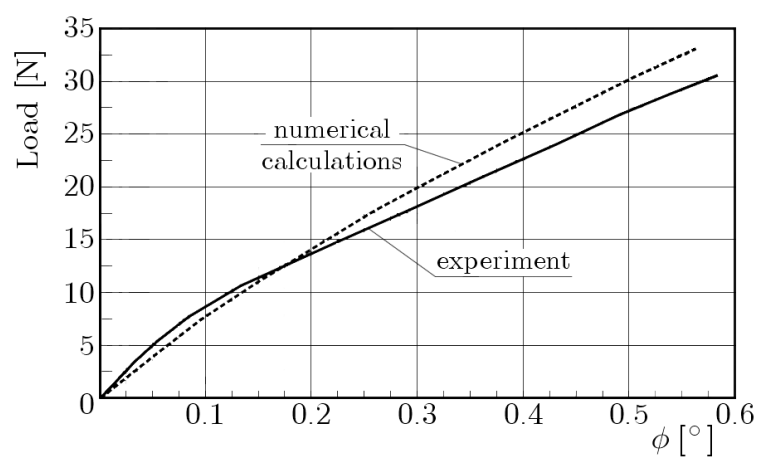

Fig. 12. A comparison of the representative equilibrium paths 
After application of appropriate corrections, the displacement distribution satisfactorily consistent with the actual one has been finally obtained (Fig. 9).

At the same time, a satisfactory conformity has been observed of the equilibrium path obtained numerically with that determined in the course of the experiment (Fig. 12). The difference in the representative parameter value has not exceed $23 \%$. Therefore, on the grounds of the rule of uniqueness of the solutions, the calculated stress distributions can be considered sufficiently reliable (Fig. 13).

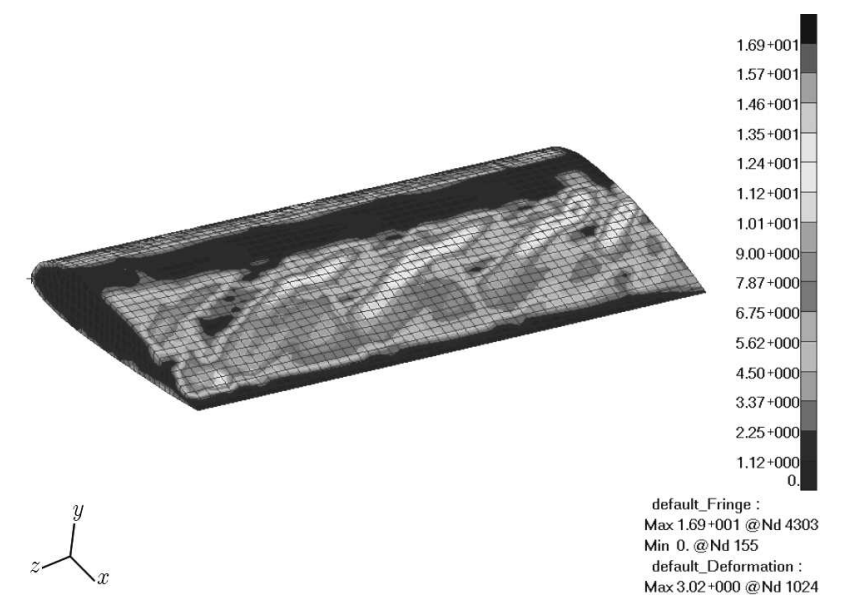

Fig. 13. Equivalent stress distributions according to $\sigma_{\max }$ hypothesis (in MPa)

\section{Summary and conclusions}

The results presented above and observations based on them should be considered in the context of a wider research program aimed at determination of properties of a series of aircraft composite skins subjected to post-critical deformation under permissible load conditions. A necessary complement to the experimental phase allowing one to obtain information about stress distribution patterns in the examined skins consists in the development of appropriate and effective FEM-based calculation models.

The satisfactory similarity found in this study between the deformations patterns and the courses of representative equilibrium paths obtained from the experiment on one hand and numerically on the other allows one to apply the rule of uniqueness of the solutions and consider the obtained stress distributions reliable. This way it can be stated that the properties of composites attributed to finite elements by PATRAN software, determined by the program based on data for individual layers of the composite, may be considered correct and corresponding to actual characteristics. However, it should be emphasised that in the case of occurrence of any defects in the real structure that may arise in the process of lamination, it is necessary to introduce an appropriate correction in the numerical model accounting for the effect of such flaws on the local stiffness of the skin.

Once a verified reference numerical model is at disposal, it is possible to apply a methodology constituting in introducing design changes to it by employing different stiffening variants. A criterion for selection of the target solution can be the highest possible value of the critical load or the lowest possible magnitude of critical deformation adopted as the representative one. The last step must consist in performing an experiment with the use of a model corresponding to the selected variant. This follows from the absolute necessity to verify the numerical model. However, this way of conduct allows one to eliminate the experimental phase involving intermediate solutions identified as failing to meet the selected criteria. 


\section{References}

1. Arborcz J., 1985, Post-buckling behavior of structures. Numerical techniques for more complicated structures, Lecture Notes in Physics, 228, USA

2. Bathe K.J., 1996, Finite Element Procedures, Prentice Hall, USA

3. BrzoskA Z., 1965, Statics and Stability of Bar and Thin-Walled Structures, PWN, Warszawa, Poland

4. Dow N.F., Hickman W.A., Rosen B.W., 1954, Data on the Compressive Strength of Skin-Stringer Panels of Various Materials, NACA TN 3064

5. Federal Aviation Administration, 2009, Composite Aircraft Structure, Advisory Circular 20-107B

6. Felippa C.A., 1976, Procedures for Computer Analysis of Large Nonlinear Structural System in Large Engineering Systems, edit. by A. Wexler, Pergamon Press, London, UK

7. Kopecki T., 2010, Advanced Deformation States In Thin-Walled Load-Bearing Structure Design Work (in Polish), Publishing House of Rzeszów University of Technology, Rzeszów, Poland

8. Kopecki T., Mazurek P., 2013, Problems of numerical bifurcation reproducing in post-critical deformation states of aircraft structures, Journal of Theoretical and Applied Mechanics, 51, 4, 969-977

9. Kopecki T., Mazurek P., 2014, Numerical representation of post-critical deformations in the processes of determining stress distributions in closed multi-segment thin-walled aircraft loadbearing structures, Maintenance and Reliability, 16, 1, 164-169

10. Marcinowski J., 1999, Nonlinear Stability of Elastic Shells, Publishing House of Technical University of Wrocław, Poland

11. Nemeth M.P., 2013,A Leonard-Sanders-Budiansky-Koiter-Type Nonlinear Shell Theory with a Hierarhy of Transverse-Shearing Deformations, NASA TP-2013-218025

12. Niu M.C., 1988, Airframe Structural Design, Conmilit Press Ltd., Hong Kong

13. Rakowski G., Kacprzyk Z., 2005,Finite Elements Method in Structure Mechanics, Publishing House of Technical University of Warszawa, Warszawa, Poland

14. Ramm E., 1987, The Riks/Wempner Approach - an Extension of the Displacement Control Method in Nonlinear Analysis, Pineridge Press, Swensea, UK

15. Ramm E., Wall W.A., 2004, Shell structures - a sensitive interrelation between physics and numerics, International Journal for Numerical Methods in Engineering, 60, 381-427

16. Seresta O., 2007, Buckling, flutter and postbuckling optimization of composite structures, PhD Thesis, Virginia State University, Blacksburg

17. TAYlor A.S., ECKFord D.J., EDIT., 1968, Aircraft Loading Actions Problems - Proceedings of a Symposium Held at Farnborough, London 